PROCEEDINGS OF THE AMERICAN MATHEMATICAL SOCIETY

Volume 125, Number 5, May 1997, Pages 1391-1397

S 0002-9939(97)03866-5

\title{
EXTREME POINTS OF UNIT BALLS IN LIPSCHITZ FUNCTION SPACES
}

\author{
RYSZARD SMARZEWSKI
}

(Communicated by Dale Alspach)

\begin{abstract}
We give a new characterization of the set ext $\left(B_{X \#}\right)$ of all extreme points of the unit ball $B_{X \#}$ in the Banach space $X^{\#}$ of all Lipschitz functions on a metric space $X$. This result is applied to get a total variation characterization of ext $\left(B_{X \#}\right)$ in the particular case when $X$ is a convex subset of a Banach space.
\end{abstract}

Let $0 \in X$ be an arbitrarily chosen point of a metric space $X=(X, d)$ which consists of at least two distinct points. Following Lindenstrauss [3] denote by $X^{\#}$ the Banach space of all functions $f: X \rightarrow \mathcal{R}$ such that $f(0)=0$ and

$$
\|f\|=\sup \left\{\frac{|f(x)-f(y)|}{d(x, y)}: x, y \in X, x \neq y\right\}<\infty .
$$

In other words, the Banach space $X^{\#}$ consists of all real-valued Lipschitz functions defined on $X$, which are equal zero at the distinguished point 0 . In the following, we always assume that the distinguished point 0 is equal to the origin of the Banach space $E$, whenever $X$ is a subset of $E$ containing the origin of $E$.

In the study of geometric Banach space theory and its various applications it is important to have a good characterization of the extreme points of unit balls. The investigation of the set of all extreme points $\operatorname{ext}\left(B_{X^{\#}}\right)$ of the unit ball $B_{X^{\#}}$ of $X^{\#}$ has been originated by Rolewicz [4] who has proved the following theorem.

Theorem A. Let $f$ be a function in $[0,1]^{\#}$ with $\|f\|=1$. Then $f \in \operatorname{ext}\left(B_{[0,1]^{\#}}\right)$ if and only if $\left|f^{\prime}(x)\right|=1$ a.e. on $[0,1]$.

Moreover, he has shown in [5] that a similar result cannot hold for the space $X=[0,1] \times[0,1]$ with Euclidean metric. Next, Cobzas [1] has characterized the extreme points in $X^{\#}$ for a rather restricted class of metric spaces $X$. Recently, Farmer [2] has presented a new characterization of the set $\operatorname{ext}\left(B_{X^{\#}}\right)$ without any additional restrictions on $X$. More precisely, he proved the following theorem.

Theorem B. Let $X$ be a metric space, and let $f$ be a function in $X^{\#}$ with the norm $\|f\|=1$. Then $f \in \operatorname{ext}\left(B_{X^{\#}}\right)$ if and only if (i) $\epsilon_{x, y}^{f}=0$ for all $x, y \in X$,

Received by the editors November 13, 1995.

1991 Mathematics Subject Classification. Primary 46B20.

Key words and phrases. Lipschitz functions, extreme points, total variation characterization.

(C)1997 American Mathematical Society 
where

$$
\begin{aligned}
\epsilon_{x, y}^{f}=\inf \left\{\epsilon>0: d\left(x_{i-1}, x_{i}\right)-\epsilon_{i} \leq\left|f\left(x_{i-1}\right)-f\left(x_{i}\right)\right|\right. & (i=1, \ldots, n), \\
x_{0} & \left.=x, x_{n}=y, \sum_{i=1}^{n} \epsilon_{i} \leq \epsilon\right\}
\end{aligned}
$$

with the infimum taken over all finite sequences $\epsilon_{1}, \ldots, \epsilon_{n}>0$ and $x_{1}, \ldots, x_{n-1} \in X$ satisfying the above inequalities.

Moreover, he noted that condition (i) is equivalent to the condition

(ii) $\epsilon_{x, 0}^{f}=0$ for every $x \in X$,

which is an immediate consequence of the triangle inequality

$$
\epsilon_{x, y}^{f} \leq \epsilon_{x, z}^{f}+\epsilon_{z, y}^{f} ; x, y, z \in X .
$$

In this paper, we first apply Theorem $\mathrm{B}$ to derive a new characterization of $\operatorname{ext}\left(B_{X \#}\right)$. Next, we use this result to obtain the following

Theorem 1. Let $X$ be a convex subset of a normed linear space $E=(E,\|\cdot\|)$, and let $f$ be a function in $X^{\#}$ such that $\|f\|=1$. Then $f \in \operatorname{ext}\left(B_{X^{\#}}\right)$ if and only if

$$
\text { (i) } \inf \left\{\sum_{i=1}^{n}\left(\left\|x_{i}-x_{i-1}\right\|-\int_{0}^{1}\left|f_{x_{i}, x_{i-1}}^{\prime}(t)\right| d t\right): x_{0}=x, x_{n}=y\right\}=0
$$

for all $x, y \in X$, where the infimum is taken over all finite sequences $x_{1}, \ldots, x_{n-1} \in$ $X$, and

$$
f_{x_{i}, x_{i-1}}(t)=f\left((1-t) x_{i-1}+t x_{i}\right), 0 \leq t \leq 1 .
$$

For this purpose, let

$$
\langle x, y\rangle=\{z \in X: d(x, y)=d(x, z)+d(z, y)\}
$$

be the metric interval with endpoints $x, y \in X$. Additionally, let $\left(x_{i}\right)_{0}^{n}$ be a metric subdivision of $\langle x, y\rangle$ with $x \neq y$, i.e., let $x_{0}=x, x_{n}=y, x_{i} \in\langle x, y\rangle, x_{i} \neq x_{j}$ for $i \neq j$, and

$$
d(x, y)=\sum_{i=1}^{n} d\left(x_{i-1}, x_{i}\right) .
$$

Then we define

$$
\rho_{f}(x, y)=\inf \left\{\|f\| d(x, y)-\sum_{i=1}^{n}\left|f\left(x_{i}\right)-f\left(x_{i-1}\right)\right|\right\},
$$

where the infimum is taken over all finite metric subdivisions $\left(x_{i}\right)_{0}^{n}$ of the interval $\langle x, y\rangle$. Additionally, we put $\rho_{f}(x, x)=0$. Since points $x_{0}=x$ and $x_{1}=y$ form a subdivision of $\langle x, y\rangle$, it follows from (3) that

$$
\rho_{f}(x, y) \leq\|f\| d(x, y)-|f(x)-f(y)|
$$

for all $x, y \in X$. Further, we have

$$
\rho_{f}(x, y) \leq\|f\| d(x, y)-\sum_{i=1}^{n+m}\left|f\left(x_{i}\right)-f\left(x_{i-1}\right)\right|
$$


for all metric subdivisions $\left(x_{i}\right)_{0}^{n}$ of $\langle x, z\rangle$ and $\left(x_{i}\right)_{n+1}^{n+m}$ of $\langle z, y\rangle$, where $z \in\langle x, y\rangle$. Hence one can take the first infimum over $x_{1}, \ldots, x_{n-1}$ and the second over $x_{n+2}, \ldots$, $x_{n+m-1}$ to get

$$
\rho_{f}(x, y) \leq \rho_{f}(x, z)+\rho_{f}(z, y), z \in\langle x, y\rangle .
$$

In general, $\rho_{f}$ does not satisfy the triangle inequality. For example, let $\|x\|_{p}(1<p$ $<\infty)$ denote $l^{p}$ - norm of $x=\left(x_{1}, x_{2}\right) \in X=\mathcal{R}^{2}$. Then we have

$$
\rho_{f}(x, y)=\|x-y\|_{p}-\left|x_{1}-y_{1}\right|
$$

for the function $f(x)=x_{1}$. Hence we get

$$
1=\rho_{f}(x, y)>\rho_{f}(x, z)+\rho_{f}(z, y)=2^{\frac{1}{p}}-1,
$$

whenever $x=(0,0), y=(0,1)$ and $z=(1,0)$.

In view of this example, we define

$$
\begin{array}{r}
\sigma_{f}(x, y)=\inf \left\{\rho_{f}\left(x, z_{1}\right)+\rho_{f}\left(z_{1}, z_{2}\right)+\ldots+\rho_{f}\left(z_{n}, y\right)\right. \\
\left.\quad z_{1}, \ldots, z_{n} \in X, n \in \mathcal{N}\right\}
\end{array}
$$

for all $x, y \in X$ and $f \in X^{\#}$. Clearly, $\sigma_{f}$ is a symmetric function such that $\sigma_{f}(x, x)=0$ and

$$
0 \leq \sigma_{f} \leq \rho_{f}
$$

In particular, this together with (4) gives

$$
|f(x)-f(y)| \leq\|f\| d(x, y)-\sigma_{f}(x, y) ; x, y \in X .
$$

Further, taking the infimum over $\left(z_{i}\right)_{1}^{n}$ and $\left(y_{i}\right)_{1}^{m}$ of the right-hand side of the inequality

$$
\begin{aligned}
\sigma_{f}(x, y) \leq & {\left[\rho_{f}\left(x, z_{1}\right)+\rho_{f}\left(z_{1}, z_{2}\right)+\cdots+\rho_{f}\left(z_{n}, z\right)\right] } \\
& +\left[\rho_{f}\left(z, y_{1}\right)+\rho_{f}\left(y_{1}, y_{2}\right)+\ldots+\rho_{f}\left(y_{m}, y\right)\right],
\end{aligned}
$$

we derive

$$
\sigma_{f}(x, y) \leq \sigma_{f}(x, z)+\sigma_{f}(z, y)
$$

and therefore

$$
\left|\sigma_{f}(x, y)-\sigma_{f}(x, z)\right| \leq \sigma_{f}(y, z)
$$

for all $x, y, z \in X$. Note also that

$$
\sigma_{f} \leq \mu \leq \rho_{f} \Longrightarrow \sigma_{f}=\mu,
$$

whenever the function $\mu: X \times X \rightarrow \mathcal{R}$ satisfies the triangle inequality on $X$. Indeed, note that

$$
\begin{gathered}
\rho_{f}\left(x, z_{1}\right)+\rho_{f}\left(z_{1}, z_{2}\right)+\ldots+\rho_{f}\left(z_{n}, y\right) \geq \mu\left(x, z_{1}\right)+\mu\left(z_{1}, z_{2}\right) \\
+\ldots+\mu\left(z_{n}, y\right) \geq \mu(x, y) \geq \sigma_{f}(x, y),
\end{gathered}
$$

and take the infimum over $\left(z_{i}\right)_{1}^{n}$ to get $\sigma_{f}=\mu$.

Theorem 2. Let $X$ be a metric space, and let $f$ be a function in $X^{\#}$ with the norm $\|f\|=1$. Then $f \in \operatorname{ext}\left(B_{X \#}\right)$ if and only if

(i) $\sigma_{f}(x, y)=0$ for all $x, y \in X$. 
Proof. Suppose first that $\sigma_{f}(x, y)=0$ for all $x, y \in X$. Moreover, take an arbitrary $\epsilon>\rho_{f}(x, y)$. Then it follows from (2) - (3) that there exists a metric subdivision $\left(x_{i}\right)_{0}^{n}$ of $\langle x, y\rangle$ for which

$$
d(x, y)-\epsilon=\left(\sum_{i=1}^{n} d_{i}\right)-\epsilon<\sum_{i=1}^{n} c_{i}
$$

where

$$
d_{i}=d\left(x_{i-1}, x_{i}\right)>0 \text { and } c_{i}=\left|f\left(x_{i}\right)-f\left(x_{i-1}\right)\right| .
$$

Since $\|f\|=1$, we have $c_{i} \leq d_{i}$. Moreover, by (11) one can find $n$ numbers $e_{i}(i=1, \ldots, n)$ such that $0 \leq e_{i}<c_{i}$ (if $\left.c_{i}>0\right), e_{i}=0$ (if $\left.c_{i}=0\right)$, and

$$
\left(\sum_{i=1}^{n} d_{i}\right)-\epsilon=\sum_{i=1}^{n} e_{i}
$$

Now denote $\epsilon_{i}=d_{i}-e_{i}$. Then we have $\epsilon_{i}>0, \sum_{i=1}^{n} \epsilon_{i}=\epsilon$, and $c_{i} \geq e_{i}=d_{i}-\epsilon_{i}$, i.e.,

$$
d\left(x_{i-1}, x_{i}\right)-\epsilon_{i} \leq\left|f\left(x_{i}\right)-f\left(x_{i-1}\right)\right|(i=1, \ldots, n) .
$$

Hence it follows from the definition of $\epsilon_{x, y}^{f}$ that $\epsilon_{x, y}^{f} \leq \epsilon$. Since $\epsilon>\rho_{f}(x, y)$ was arbitrary, we conclude that

$$
0=\sigma_{f}(x, y) \leq \epsilon_{x, y}^{f} \leq \rho_{f}(x, y)
$$

for all $x, y \in X$. This in conjunction with (1) enables to apply (10) in order to get $\epsilon_{x, y}^{f}=\sigma_{f}(x, y)=0$. Thus Theorem B yields $f \in \operatorname{ext}\left(B_{X^{\#}}\right)$, which completes the proof of necessity.

For the proof of sufficiency, suppose that there exist $f \in X^{\#}$ and $z \in X$ for which $\|f\|=1$ and $Y=\left\{y: \sigma_{f}(z, y)>0\right\} \neq \emptyset$. Then the triangle inequality and symmetry of $\sigma_{f}$ yield

$$
\sigma_{f}(x, y)=\sigma_{f}(z, y)
$$

for all $x \in X \backslash Y$ and $y \in Y$. This together with (8) and (9) enables to repeat mutatis mutandis Farmer's proof [2] of sufficiency of Theorem B, with $\epsilon_{x, y}^{f}$ replaced by $\sigma_{f}(x, y)$, in order to show that $f \notin \operatorname{ext}\left(B_{X^{\#}}\right)$.

From now on, we will assume that $X$ is a convex subset of a normed linear space $(E,\|\cdot\|)$. In this case, we define

$$
\hat{\rho}_{f}(x, y)=\inf \left\{\|f\|\|x-y\|-\sum_{i=1}^{n}\left|f\left(x_{i}\right)-f\left(x_{i-1}\right)\right|\right\},
$$

where the infimum is taken only over all finite subdivisions $\left(x_{i}\right)_{0}^{n}$ of the form

$$
x_{i}=\left(1-t_{i}\right) x+t_{i} y\left(0=t_{0}<t_{1}<\ldots<t_{n}=1\right) .
$$

It is clear that (2) holds for these algebraic subdivisions of the algebraic interval

$$
[x, y]=\{(1-t) x+t y: 0 \leq t \leq 1\}
$$

and that $[x, y]=\langle x, y\rangle$ and $\hat{\rho}_{f}(x, y)=\rho_{f}(x, y)$ for all $x, y \in X$, whenever $E$ is a strictly convex space. In general, we have only $\rho_{f} \leq \hat{\rho}_{f}$.

If $\hat{\sigma}_{f}(x, y)$ is defined by formula (6) with $\rho_{f}$ replaced by $\hat{\rho}_{f}$, then $\sigma_{f} \leq \hat{\sigma}_{f}$. By the same arguments as above, one can also prove that $\hat{\rho}_{f}$ and $\hat{\sigma}_{f}$ satisfy inequality 
(4) and the triangle inequality, respectively. In particular, by using (2) and (4) we obtain

$$
\hat{\sigma}_{f}(x, y) \leq \hat{\rho}_{f}\left(x_{0}, x_{1}\right)+\ldots+\hat{\rho}_{f}\left(x_{n-1}, x_{n}\right) \leq\|f\|\|x-y\|-\sum_{i=1}^{n}\left|f\left(x_{i}\right)-f\left(x_{i-1}\right)\right|
$$

for all metric subdivisions $\left(x_{i}\right)_{0}^{n}$ of $\langle x, y\rangle$. Hence we derive $\hat{\sigma}_{f} \leq \rho_{f}$. Therefore, one can apply (10) with $\mu=\hat{\sigma}_{f}$ in order to get $\hat{\sigma}_{f}=\sigma_{f}$.

Lemma 1. Let $X$ be a convex subset of a normed linear space $E=(E,\|\cdot\|)$, and let $f \in X^{\#}$. Then we have

$$
\hat{\rho}_{f}(x, y)=\|f\|\|x-y\|-V_{0}^{1}\left(f_{x, y}\right)=\|f\|\|x-y\|-\int_{0}^{1}\left|f_{x, y}^{\prime}(t)\right| d t,
$$

where $V_{0}^{1}\left(f_{x, y}\right)$ denotes the total variation of the function $f_{x, y}$ defined by

$$
f_{x, y}(t)=f((1-t) x+t y)(0 \leq t \leq 1) .
$$

Proof. By (13) we obtain

$$
\begin{aligned}
\hat{\rho}_{f}(x, y) & =\|f\|\|x-y\|-\sup \left\{\sum_{i=1}^{n}\left|f\left(x_{i}\right)-f\left(x_{i-1}\right)\right|\right\} \\
& =\|f\|\|x-y\|-V_{0}^{1}\left(f_{x, y}\right),
\end{aligned}
$$

where the supremum is taken over all finite algebraic subdivisions $\left(x_{i}\right)_{0}^{n}$ of $[x, y]$. Since $f \in X^{\#}$, we have

$$
\left|f_{x, y}(t)-f_{x, y}(s)\right| \leq\|f\|\|x-y\||t-s|(0 \leq t, s \leq 1) .
$$

Hence the derivative $f_{x, y}^{\prime}(t)$ exists almost everywhere on $[0,1]$, and the function $t \rightarrow f_{x, y}^{\prime}(t)$ is integrable. Moreover, we have

$$
V_{0}^{1}\left(f_{x, y}\right)=\int_{0}^{1}\left|f_{x, y}^{\prime}(t)\right| d t .
$$

This in conjunction with (14) completes the proof.

In view of the fact that $\hat{\sigma}_{f}=\sigma_{f}$, Theorem 1 is an immediate consequence of Lemma 1 and Theorem 2. Moreover, it follows from the triangle inequality for $\sigma_{f}$ that Theorems 1 and 2 remain true, whenever we put either $x=0$ or $y=0$ into them. In particular, if the interval $X=[0,1]$ is equipped with the metric $d(x, y)=|x-y|$, then Lemma 1 yields

$$
\rho_{f}(x, y)=\hat{\rho}_{f}(x, y)=|x-y|\left(1-\int_{0}^{1}\left|f^{\prime}(s)\right| d s\right)
$$

for all $x, y \in[0,1]$. On the other hand, by (5) and (7) one can apply (10) with $\mu=\rho_{f}$ to get $\sigma_{f}=\rho_{f}$. Hence Theorem A follows directly from Theorem 2 .

Finally, we present another application of Theorem 2 which shows that the set $\operatorname{ext}\left(B_{X \#}\right)$ of all extreme points of the unit ball $B_{X \#}$ of $X^{\#}$ is quite rich, whenever 
$X$ is a normed linear space. For this purpose, denote by $X^{*}$ the dual space of $X$, and note that

$$
\sigma_{f}(x, x+\alpha z)=\sigma_{f}(0, \alpha z)=|\alpha| \sigma_{f}(0, z)(\alpha \in \mathcal{R} ; x, z \in X)
$$

for every functional $f \in X^{*}$. To prove these identities, we need only to change variables $z_{k} \rightarrow z_{k}+x \quad\left(z_{k} \rightarrow \alpha z_{k}\right)$ in the definition of $\hat{\sigma}_{f}=\sigma_{f}$ applied to $y=$ $x+\alpha z(y=\alpha z$, respectively), and use the identity

$$
\hat{\rho}_{f}(x, y)=\|f\|\|x-y\|-|f(x-y)|(x, y \in X),
$$

which is a direct consequence of Lemma 1 and linearity of $f$. Since $\sigma_{f}$ satisfies the triangle inequality, it follows from (15) that

$$
\sigma_{f}\left(0, z_{1}+z_{2}\right) \leq \sigma_{f}\left(0, z_{1}\right)+\sigma_{f}\left(z_{1}, z_{1}+z_{2}\right)=\sigma_{f}\left(0, z_{1}\right)+\sigma_{f}\left(0, z_{2}\right) .
$$

This in conjunction with (15) means that the function $z \rightarrow \sigma_{f}(0, z)(z \in X)$ is a seminorm on $X$.

Theorem 3. Let $X$ be a normed linear space. Then we have

$$
\operatorname{ext}\left(B_{X^{\#}}\right) \cap X^{*}=\operatorname{ext}\left(B_{X^{*}}\right) .
$$

Proof. In view of definition of extreme points, we directly have

$$
\operatorname{ext}\left(B_{X^{\#}}\right) \cap X^{*} \subset \operatorname{ext}\left(B_{X^{*}}\right) .
$$

Conversely, let a functional $f \in X^{*}$ be such that $\|f\|=1$ and $f \notin \operatorname{ext}\left(B_{X^{\#}}\right)$. We need only to prove that $f \notin \operatorname{ext}\left(B_{X^{*}}\right)$. By Theorem 2 the set

$$
Y=\left\{y: \sigma_{f}(0, y)>0\right\}
$$

is nonempty. Moreover, it follows from (15) that the set $X \backslash Y$ is a linear subspace of $X$ which, in view of (12), has the property

$$
\sigma_{f}(x, y)=\sigma_{f}(0, y)(x \in X \backslash Y, y \in Y) \text {. }
$$

Now take a point $y_{0} \in Y$, and denote by $X_{0}$ the linear subspace spanned by $y_{0}$ and $X \backslash Y$. Next, define the linear functional $g$ on $X_{0}$ by the formula

$$
g\left(x+\alpha y_{0}\right)=\alpha \sigma_{f}\left(0, y_{0}\right)(x \in X \backslash Y, \alpha \in \mathcal{R}) .
$$

Then it follows from (15) and (16) that

$$
\left|g\left(x+\alpha y_{0}\right)\right|=\sigma_{f}\left(x, x+\alpha y_{0}\right)=\sigma_{f}\left(0, x+\alpha y_{0}\right)(x \in X \backslash Y, \alpha \in \mathcal{R}),
$$

whenever $x+\alpha y_{0} \in Y$. Otherwise, if $x+\alpha y_{0} \notin Y$ then $\alpha=0$ and (17) is obvious. Since the function $z \rightarrow \sigma_{f}(0, z)$ is a seminorm on $X$ and $g$ satisfies condition (17) on $X_{0}$, it follows from the Hahn-Banach theorem that the functional $g: X_{0} \rightarrow \mathcal{R}$ has an extension to the whole space $X$, which satisfies the inequality

$$
|g(z)| \leq \sigma_{f}(0, z), z \in X
$$

Consequently, one can apply (4) and (7) to get

$$
|g(z)| \leq\|z\|-|f(z)|, z \in X .
$$

Thus $g \in X^{*}$ and $f_{k} \in X^{*}(k=1,2)$, where functionals $f_{k}$ are defined by

$$
f_{k}(z)=f(z)+(-1)^{k} g(z) \text {. }
$$

Therefore, we obtain

$$
\left|f_{k}(z)\right| \leq|f(z)|+|g(z)| \leq\|z\|
$$


for every $z \in X$. Hence we have $\left\|f_{k}\right\| \leq 1(k=1,2)$ and $f_{k}\left(y_{0}\right) \neq f\left(y_{0}\right)$, which in conjunction with the identity $f=\left(f_{1}+f_{2}\right) / 2$ shows that $f \notin \operatorname{ext}\left(B_{X^{*}}\right)$. Thus the proof is completed.

\section{REFERENCES}

1. S. Cobzas, Extreme points in Banach spaces of Lipschitz functions, Mathematica 31 (54) (1989), 25-33. MR 91j:46032

2. J.D. Farmer, Extreme points of the unit ball of the space of Lipschitz functions, Proc. Amer. Math. Soc. 121 (1994), 807-813. MR 94i:46038

3. J. Lindenstrauss, On nonlinear projections in Banach spaces, Michigan Math. J. 11 (1964), 263-287. MR 29:5088

4. S. Rolewicz, On extremal points of the unit ball in the Banach space of Lipschitz continuous functions, J. Austral. Math. Soc. Ser. A 41 (1986), 95-98. MR 87k:46022

5. S. Rolewicz, On optimal observability of Lipschitz systems, Selected Topics in Operations Research and Mathematical Economics, Lecture Notes in Econom. and Math. Systems, vol. 226, Springer-Verlag, Berlin, 1984, pp. 151-158. MR 86a:49076

Department of Mathematics, M. Curie-Sklodowska University, 20-031 Lublin, Poland Current address: Institute of Mathematics, Catholic University of Lublin, Al. Raclawickie 14, 20-950 Lublin, Poland

E-mail address: smarz@golem.umcs.lublin.pl 